\title{
Stromal disrupting effects of nab-paclitaxel in pancreatic cancer
}

\author{
R Alvarez¹, M Musteanu², E Garcia-Garcia ${ }^{2}$, P P Lopez-Casas², D Megias², C Guerra², M Muñoz ${ }^{2}$, Y Quijano ${ }^{1}$, \\ A Cubillo ${ }^{1}$, J Rodriguez-Pascual ${ }^{1}$, C Plaza ${ }^{1}$, E de Vicente ${ }^{1}$, S Prados ${ }^{1}$, S Tabernero ${ }^{1}$, M Barbacid ${ }^{2}$, F Lopez-Rios ${ }^{1}$ \\ and M Hidalgo ${ }^{*} 1,2$ \\ ${ }^{1}$ Centro Integral Oncológico Clara Campal (CIOCC), Oña 10, 28050 Madrid, Spain and ${ }^{2}$ Spanish National Cancer Research Centre \\ (CNIO), Melchor Fernández Almagro 3, 28029 Madrid, Spain
}

Background: Nab-paclitaxel and gemcitabine have demonstrated a survival benefit over gemcitabine alone in advanced pancreatic cancer (PDA). This study aimed to investigate the clinical, biological, and imaging effects of the regimen in patients with operable PDA.

Methods: Patients with operable PDA received two cycles of nab-paclitaxel and gemcitabine before surgical resection. FDG-PET and CA19.9 tumour marker levels were used to measure clinical activity. Effects on tumour stroma were determined by endoscopic ultrasound (EUS) elastography. The collagen content and architecture as well as density of cancer-associated fibroblasts (CAFs) were determined in the resected surgical specimen and compared with a group of untreated and treated with conventional chemoradiation therapy controls. A co-clinical study in a mouse model of PDA was conducted to differentiate between the effects of nab-paclitaxel and gemcitabine.

Results: A total of 16 patients were enrolled. Treatment resulted in significant antitumour effects with $50 \%$ of patients achieving a $>75 \%$ decrease in circulating CA19.9 tumour marker and a response by FDG-PET. There was also a significant decrement in tumour stiffness as measured by EUS elastography. Seven of 12 patients who completed treatment and were operated had major pathological regressions. Analysis of residual tumours showed a marked disorganised collagen with a very low density of CAF, which was not observed in the untreated or conventionally treated control groups. The preclinical co-clinical study showed that these effects were specific of nab-paclitaxel and not gemcitabine.

Conclusion: These data suggest that nab-paclitaxel and gemcitabine decreases CAF content inducing a marked alteration in cancer stroma that results in tumour softening. This regimen should be studied in patients with operable PDA.

The outcome of patients with PDA remains poor (Hidalgo, 2010). One important characteristic of this disease is the formation of a rich stromal reaction that accompanies the malignant transformed epithelium (Mahadevan and Von Hoff, 2007). The stroma is composed of extracellular matrix proteins such as collagen, hyaluronic acid, and SPARC (secreted protein acidic and rich in cysteine) and cellular elements such as cancer-associated fibroblast (CAF), endothelial, immune, and inflammatory cells (Mahadevan and Von Hoff, 2007). The stroma in PDA contributes to poor vascularisation and high intratumoural pressure that decreases drug diffusion (Von Hoff et al, 2009). There is an increasing interest in targeting different components of the cancer stroma in PDA and several preclinical studies using genetically engineered mouse models (GEMMs) of PDA show promising results (Olive et al, 2009; Provenzano et al, 2012; Jacobetz et al, 2013). The elimination of the cancer stroma leads to decrease intratumour pressure, heighted vascularisation, and improved drug delivery.

One interesting stromal target of PDA is SPARC, which is overexpressed in PDA (Infante et al, 2007). Although the functions of this protein are not fully understood, it is implicated in stromal

*Correspondence: Dr M Hidalgo; E-mail: mhidalgo@cnio.es

Received 25 February 2013; revised 26 June 2013; accepted 1 July 2013; published online 1 August 2013

(c) 2013 Cancer Research UK. All rights reserved 0007-0920/13 
formation and trafficking, invasion and metastasis, and has been linked to a worse prognosis (Infante et al, 2007; Arnold et al, 2010). SPARC binds albumin leading to the hypothesis that it may facilitate the delivery to the tumour of albumin-bound therapeutics. A phase 2 clinical trial of nab-paclitaxel in combination with gemcitabine in patients with advanced PDA showed significant antitumour activity with an objective response rate of $48 \%$, median survival of 12.2 months, and 1-year survival of $48 \%$ (Von Hoff et al, 2011). A recently reported phase 3 study has shown improvement in survival with nab-paclitaxel and gemcitabine, as compared with gemcitabine in patients with advanced PDA. In a parallel preclinical study using patient-derived xenograft models of PDA, we showed that exposure to nab-paclitaxel collapsed the PDA stroma increasing the intratumour concentration of gemcitabine by approximately three-fold (Von Hoff et al, 2011). A subsequent study in GEMM of PDA indicated that nabpaclitaxel increases the intratumour concentration of gemcitabine by inactivation of the cytidine deaminase, an enzyme involved in the catabolism of gemcitabine (Frese et al, 2012).

This study aimed to determine the clinical, biological, and imaging effects of nab-paclitaxel and gemcitabine in patients with operable PDA.

\section{MATERIALS AND METHODS}

Clinical trial eligibility criteria. Patients with histological or cytological proven PDA with resectable or borderline resectable disease were eligible. Other eligibility criteria included: age $\geqslant 18$ years old; no prior treatment for PDA; Eastern Cooperative Oncology Group performance status 0 or 1 ; adequate renal (creatinine $<0.6 \mathrm{mg} \mathrm{dl}^{-1}$ ), liver (bilirubin $<2 \mathrm{mg} \mathrm{dl}^{-1}$, AST and ALT $<1.5 \times$ upper limit of normal); and bone marrow (ANC $>1.5 \times 10^{9}$ cells $\mu \mathrm{l}^{-1}$, platelets $>100000$ cells $\mu \mathrm{l}^{-1}$, haemoglobin $>9 \mathrm{~g} \mathrm{dl}^{-1}$ ) function. All patients provided written informed consent. The study was approved by the institutional review board.

Study design, evaluations, and treatment. Before study entry, patients were studied with a complete medical history and physical exam; complete blood counts and serum chemistry; CA19.9 tumour marker; high-resolution CT scan of the abdomen, FDGPET scan; and endoscopic ultrasound (EUS) elastography. Treatment consisted of nab-paclitaxel $125 \mathrm{mg} \mathrm{m}^{-2}$ followed by gemcitabine $1000 \mathrm{mg} \mathrm{m}^{-2}$ administered on days 1,8 , and 15 , every 28 days for two cycles. Treatment-related toxicities were evaluated by National Cancer Institute Common Terminology Criteria of Adverse Events version 3.0. A CT scan, FDG-PET, elastography, and CA19.9 were used to determine tumour response after two cycles of treatment. Objective tumour responses were evaluated according to RECIST version 1.1 criteria (Eisenhauer et al, 2009). EUS elastography was performed with the linear Pentax EUS (Montvale, NJ, USA) and the Hitachi EUB900 equipment (Tokyo, Japan). Representative areas of the tumour and surrounding normal tissues were selected for analysis. The results of the elastography evaluation was defined by the quotient strain ratio between the normal and tumour regions as previously described (Iglesias-Garcia et al, 2010). Patients with stable or responding tumours were schedule for surgical resection 6-8 weeks after the last chemotherapy dose. The degree of tumour regression in the surgical specimen was determined adapting the Ryan's classification utilised in rectal cancer including four categories: GRT-0, complete response; GRT-1, important partial response with only single cells or isolated cell groups left; GRT-2, partial response but large residual tumour, and GRT-3 low or no response and large residual cancer (Ryan et al, 2005). R1 resection was considered if there were tumour cells present $\leqslant 1 \mathrm{~mm}$ of the resection margin. Postoperative treatment consisted in four cycles of standard dose of gemcitabine.
Preclinical study. The compound K-Ras ${ }^{+/ L S L G 12 V g e o} ; p 53^{\text {flox/flox }}$; Elas-tTA/tetO-Cre mice used in this study were kindly provided by Dr Mariano Barbacid (Guerra et al, 2011). Mice were treated with nab-paclitaxel at a final concentration of $50 \mathrm{mg} \mathrm{kg}^{-1}$ paclitaxel I.V. once a week and gemcitabine at a final concentration of $50 \mathrm{mg} \mathrm{kg}^{-1}$ I.P. twice a week for 3 weeks. Tumour growth was monitored in vivo by high-resolution ultrasound. Average tumour volumes at baseline in the nab-paclitaxel, gemcitabine, and combination groups were 20.8 (4.7), 113.3 (39.3), and $87.9(34.1) \mathrm{mm}^{3}$, respectively. Responses upon chemotherapy were evaluated as follow: progression, when tumour volumes at the end of the study were $>50 \%$ larger than at the start point; partial response, when tumour volumes regressed by $>50 \%$ when compared with initial volumes and complete response, and when tumours at the end of the study could not be detected by ultrasound. All experiments were approved by the CNIO Ethical Committee and performed in accordance with the guidelines for Ethical Conduct in the Care and Use of Animals as stated in the International Guiding Principles for Biomedical Research Involving Animals, developed by the Council for International Organizations of Medical Sciences.

Tissue assessments. The primary end point of the study was to determine the effects of treatment on tumour stroma including assessment of collagen content and structure and CAFs. In addition to study samples obtained from patients and mouse models, we also analysed a set of six samples from patients with untreated PDA and six samples from patients treated with conventional chemotherapy and radiation therapy. Collagen analysis was assessed using collagen I primary rabbit antibody ( 1 : 100; Rockland Immunochemicals, Gilbertsville, PA, USA) for $1 \mathrm{~h}$ at $37^{\circ} \mathrm{C}$ and as secondary antibody Alexa Fluor 555 donkey anti-rabbit IgG (1:200; Invitrogen, Paisley, UK). Activated fibroblasts were determined with double staining using vimentin, as total fibroblasts marker (1:50; Cell Signaling Technology, Danvers, MA, USA) and smooth muscle actin (SMA) as an activated fibroblast marker (1:100; DakoCytomation, Glostrup, Denmark). The secondary antibodies used were Alexa Fluor 488 donkey anti-rabbit IgG $(1: 200$; Invitrogen) for vimentin and Alexa Fluor 555 donkey anti-mouse IgG (1:200; Invitrogen) for SMA. Sections were counterstained with DAPI (Invitrogen) and mounted with Prolong Gold Antifade reagent (Invitrogen). Samples were acquired using a laser scanning confocal microscope TCS-SP5 (AOBS) Leica (Leica Microsystems, Wetzlar, Germany) with two magnifications $\times 20 \mathrm{HCX}$ PL APO CS $(0.7 \mathrm{NA})$ and $\times 40 \mathrm{HCX}$ PL APO CS (1.25 NA) oil immersion objectives. Acquisition Software was LAS AF v.2.4.1 (Leica Microsystems). Fibroblast quantification was performed with Definiens Developer XD Software (Munich, Germany). Detected cells were classified depending on the ratio between mean layer intensities of both, vimentin and SMA. Activated fibroblasts were considered when they co-expressed SMA and vimentin. We determined the fibroblast density index by adjusting the number of cells counted to the proportion of tumour fibrosis observed in the specimen (adapted from Erkan et al, 2008). The quantification was done in two different and representative fields (20 steps) for each sample. In the preclinical study, immunohistochemistry was performed using antibodies against mouse SPARC (R\&D Systems, MAB942, Minneapolis, MN, USA) and cleaved caspase 3 (Cell Signaling Technology, 9661).

Statistical analysis. The analysis was made by 'intention to treat' with all patients included using the IBM SPSS Statistics Version 20.0 (IBM, Madrid, Spain). Quantitative variables pre- and posttreatment were compared using ANOVA test, Student's $t$-test, and non-parametric Mann-Whitney $U$-test and Wilcoxon test for two related samples. The correlation between the outcome variables was tested with the Spearman correlation coefficient. In the preclinical study, all values are represented as mean \pm s.e.m. Immunohistochemistry quantifications were evaluated for significance using the 
unpaired $t$-test with a $P$-value below 0.05 considered significant for all analyses. Significant differences between experimental groups were: ${ }^{\star} P<0.05$ or ${ }^{* *} P<0.01$.

\section{RESULTS}

Clinical activity of nab-paclitaxel and gemcitabine in PDA. A total of 16 patients, whose principal characteristics are listed in Table 1, received two cycles of nab-paclitaxel and gemcitabine before surgical resection using the previously published dose and schedule (Von Hoff et al, 2011). Three patients developed disease progression during the preoperative treatment and were not resected. One additional patient with potentially resectable cancer was not operated because of high surgical risk. A total of 12 patients underwent surgery. Overall treatment was well tolerated with side effects in the range of what has been previously reported.

Figure 1 shows the results of the main clinical parameters studied. The mean CA19.9 level decreased from $2588 \mathrm{U} \mathrm{dl}^{-1}$ pretreatment to $1056 \mathrm{U} \mathrm{dl}^{-1}$ post-treatment $(P=0.001)$ with $50 \%$ of patients having a $>75 \%$ reduction. Similarly, the max standardised uptake value (SUV) of FDG decreased from a mean of 7.2 (range, 2.98-13.4) before treatment to 4.5 (range, 1.9-9) after treatment $(P=0.005)$, including eight $(50 \%)$ of the patients with a partial metabolic response by FDG-PET (Supplementary Figure 1 shows a representative case). There was a statistically significant correlation between improvement in CA19.9 tumour marker and FDG PET response $(P=0.05)$. However, no objective responses were observed. We also determined tumour stiffness by elastography. The ratio value, a measurement of tumour stiffness, diminished from a value 36 pre-treatment to 18 post-treatment $(P=0.002)$. These data suggest that tumours became softener after treatment. There was a statistically significant correlation between changes from baseline in elastography ratio and CA19.9 response $(P=0.019)$.

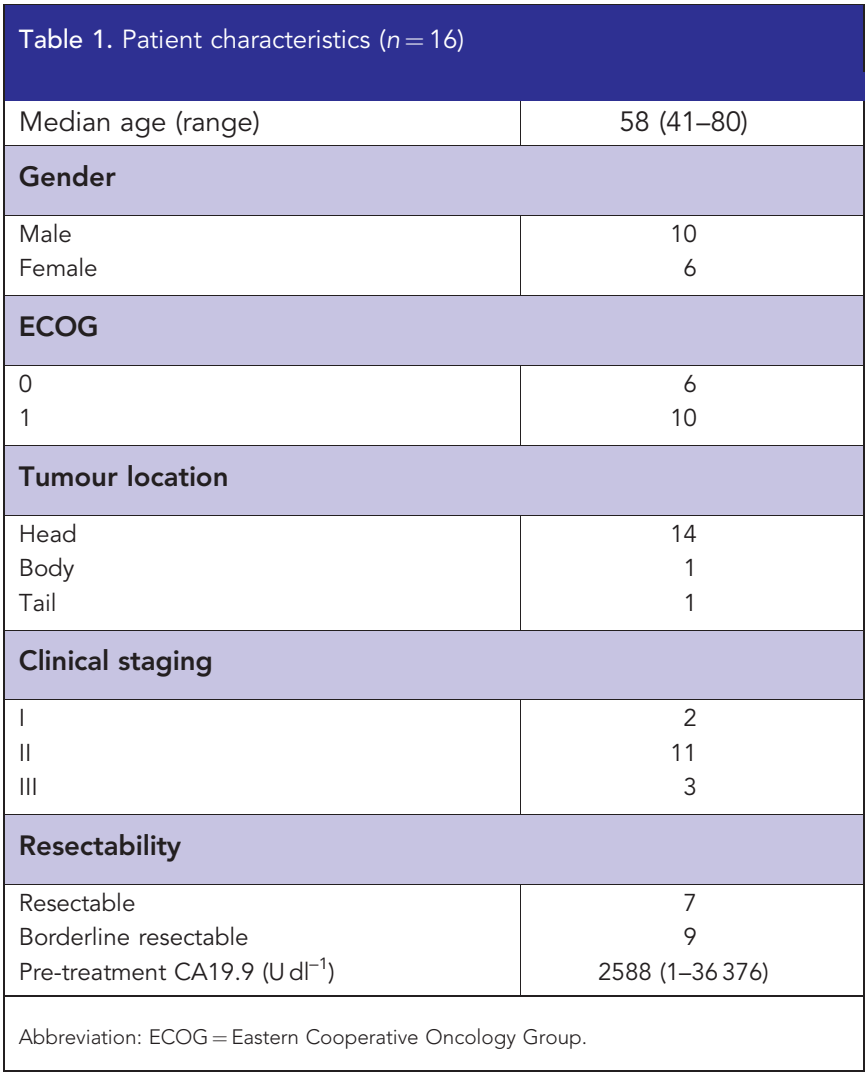

Of the 12 patients operated, 11 had $\mathrm{R} 0$ resection and 1 patient had R1 resection. In the final pathological analysis, two cases were neuroendocrine carcinomas and 10 were pancreatic adenocarcinoma. Of the 10 patients with PDA, 1 had a complete pathological response, 6 had major pathological responses (GRT-1), 1 had a partial response (GRT-2) and 2 did not response (GRT-3). Supplementary Figure 1 shows pathological analysis of a representative patient.

Tissue effects of nab-paclitaxel and gemcitabine. Nine of the 10 patients with PDA had SPARC-positive tumours. In the subset of patients with SPARC expression in the tumour stroma, there was no correlation between the degree of SPARC expression and clinical or pathological responses. As shown in Figure 2A, type I collagen analysis of tumour tissue samples from patients treated with nab-paclitaxel plus gemcitabine revealed a less abundant fibrillar collagen matrix around tumour glands. In areas of tumour regressions the collagen had an amorphous structure, with a discontinuous and disorganised network of type I collagen fibres. This is in contrast with samples of untreated PDA used as control that showed long, continuous well-organised, and imbricated fibres. Similarly, in tumours treated with conventional chemoradiation studied for comparison, there was an increased in collagen matrix with dense bundles of organised collagen around tumour glands.

In addition, patients treated with nab-paclitaxel plus gemcitabine had a lower number of CAF. The average number of CAF in the nab-paclitaxel plus gemcitabine-treated group was $267 \pm 179$ while samples from untreated patients and samples from patients treated with conventional chemotherapy and radiation therapy was $642 \pm 378(P=0.01)$ and $557 \pm 140(P=0.037)$. Interestingly, the proportion of SMA + fibroblasts was similar in all these cases ranging from 76 to $78 \%$ (Figure $2 \mathrm{~B}$ and $\mathrm{C}$ ).

As the proportion of tumour stroma changed from one setting to another, we calculated the CAF density index on these samples. As shown in Figure 2D, the CAF density in untreated PDA tumours was $15 \pm 10$. In cases treated with conventional chemoradiation, because the stroma content increased the density decreased to $12 \pm 11$. In nab-paclitaxel plus gemcitabine-treated cases, this number decreased to $6 \pm 3$ because of higher stromal content and decrease number of CAF.

Preclinical study in GEMM of PDA. In order to determine the specific contributions of nab-paclitaxel and gemcitabine to these results, we performed an in vivo study in GEMM of PDA (Guerra et al, 2011). As shown in Supplementary Figure 2, the cancer, induced in these models by activation of KRAS and inactivation of TP53, is SPARC + in CAF. Mice were enrolled in this study as individual subjects when tumours were detected by two consecutive ultrasound measurements. As expected, the combination of nab-paclitaxel and gemcitabine was very effective and resulted in a complete tumour regression in two mice and a partial tumour regressions in one mouse, as determined by ultrasound after 21 days of treatment (Figure 3A). Gemcitabine alone treatment resulted in minor responses, while nab-paclitaxel alone did not show any antitumour activity (Figure 3A). Consistently, nabpaclitaxel plus gemcitabine significantly increased the proportion of apoptotic cells compared with control (Figure $3 \mathrm{~B}$ and $\mathrm{C}$ ). Average increase in tumour volumes in the nab-paclitaxel, gemcitabine, and combination groups on day 21 compare with baseline were $14.1,4.6$, and 0.96 , respectively.

Analysis of type I collagen revealed a decrease in collagen matrix in nab-paclitaxel and nab-paclitaxel plus gemcitabine groups compared with control and gemcitabine groups (Figure 4A). Type I collagen matrix showed a heterogeneous pattern alternating between areas with moderate extracellular matrix and areas with very low collagen matrix. Collagen bundles were composed of disrupted and disorganised fibres. However, control and 

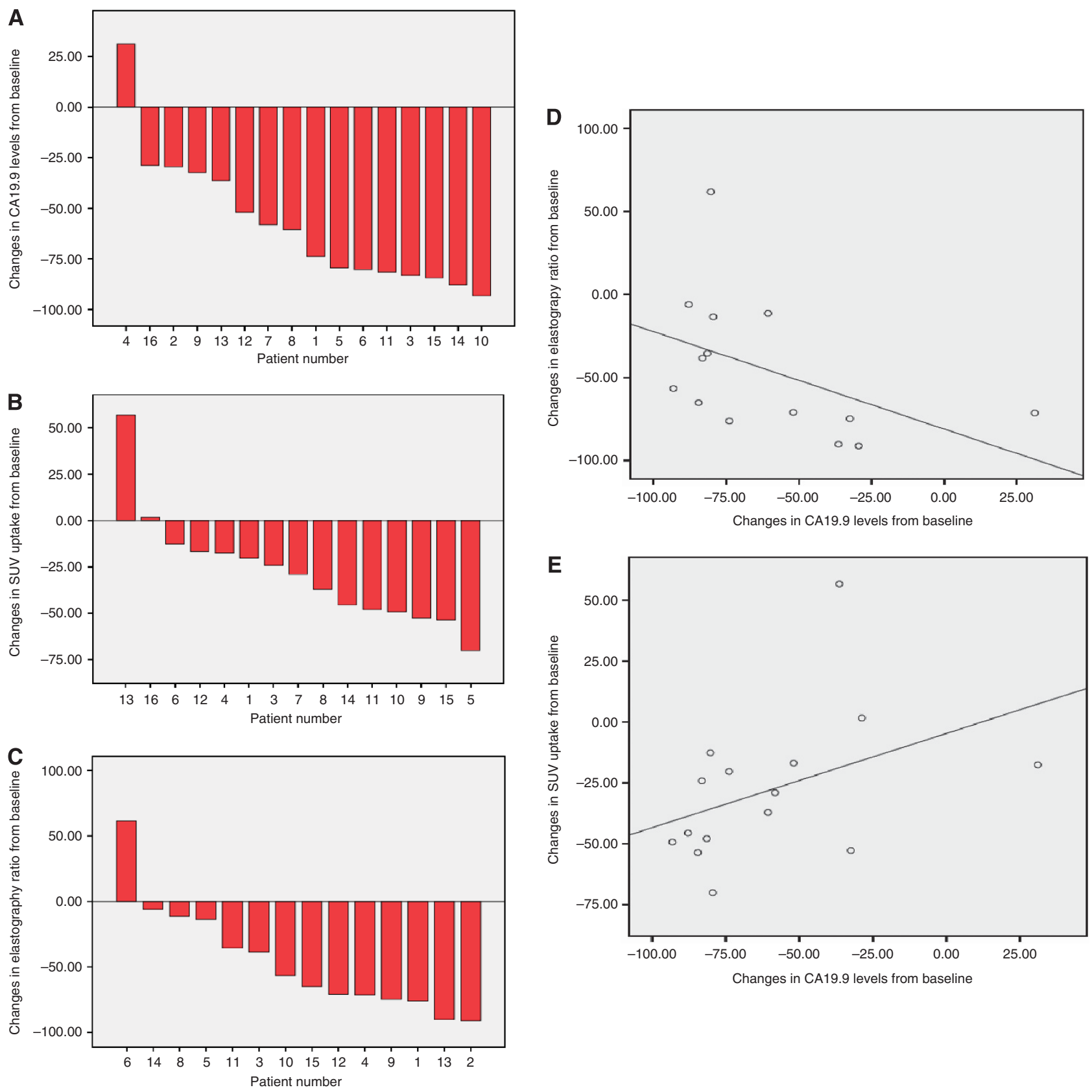

Changes in CA19.9 levels from baseline

Figure 1. Antitumour effects of nab-paclitaxel and gemcitabine in PDA. Panels (A-C) show waterfall plots of changes from baseline variation in the levels of the CA19.9 tumour marker, SUV FDG uptake and elastrography strain ratio of patients treated with nab-paclitaxel and gemcitabine. Studies were performed at baseline, before treatment, and after two 4 weeks cycles of treatment and best response is shown. As depicted, in all patients but one, there was evidence of antitumour effects, which was marked in more than half of the patients. Panels (D) and (E) show a scatter plot graph of the change from baseline in FDG SUV and elastography strain ratio with CA19.9 levels, respectively, which is an accepted parameter of clinical efficacy. As shown, improvement in both imaging parameters were correlated with improvement in CA19.9 with a $P=0.05$ and 0.01 , respectively.

gemcitabine groups showed a dense collagen matrix with long and imbricated collagen fibres around tumour glands (Figure 4A). The total number of CAF in nab-paclitaxel group and nab-paclitaxel plus gemcitabine group was $37 \pm 17.4$ and $35 \pm 24.5$, respectively, with $68 \%$ of SMA + fibroblasts in both treated groups (Figure $4 \mathrm{~B}$ and $\mathrm{C})$. The total number of CAF in control group was similar to the former groups, $39 \pm 18.4$ and $69 \%$ of SMA + fibroblasts. However, the gemcitabine-treated group showed a higher number of fibroblasts, $173 \pm 125.2$ than the other groups with a similar proportion of SMA $+(65.2 \%)$. The CAF density ranged between 2.8 and 2.3 in nab-paclitaxel and nab-paclitaxel plus gemcitabine group and this parameter increased to 7 in the gemcitabine group; however, because low number of cases, there were no statistically significant differences.

\section{DISCUSSION}

This study aimed to investigate the effects of nab-paclitaxel and gemcitabine in PDA stroma. To this end, we conducted a clinical and co-clinical study in patients with operable PDA and in 
A

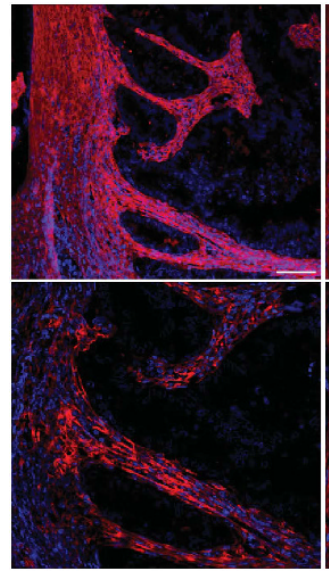

Untreated

B

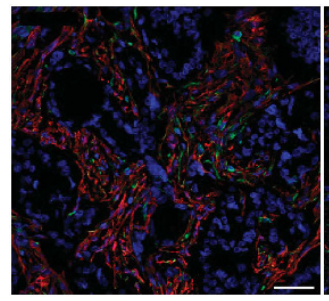

Untreated

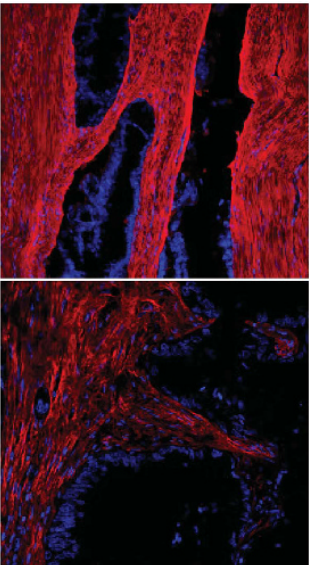

Chemoradiation

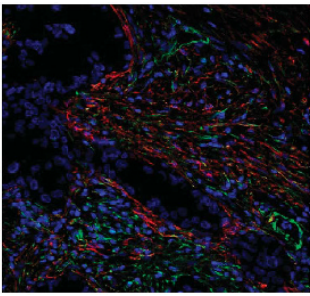

Chemoradiation

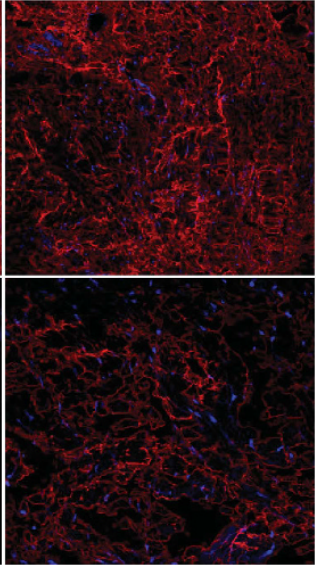

Nab-paclitaxel + gemcitabine

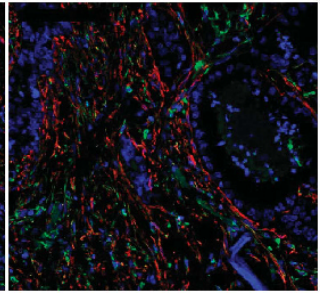

Nab-paclitaxel +
C

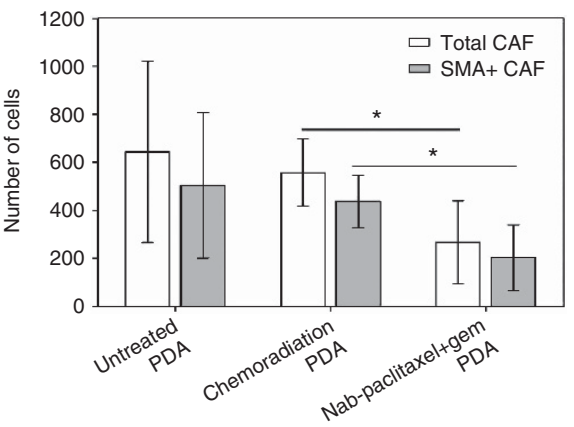

D

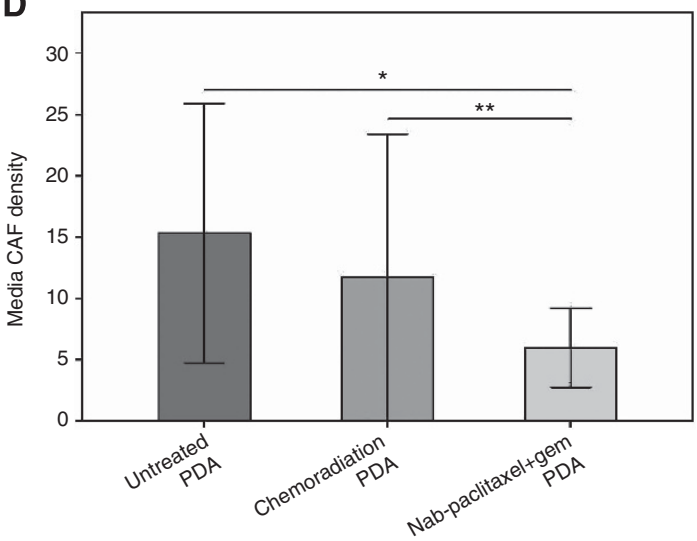

Figure 2. Tissue effects of nab-paclitaxel and gemcitabine in human PDA. Panel (A) shows confocal microscopy analysis of type I collagen immunofluorescence as main component of extracellular matrix in untreated sample, a sample of a patient treated with chemoradiation, and a sample of a patient treated with nab-paclitaxel and gemcitabine $(\times 20$, top, and the same field at $\times 40$, bottom; scale bar $=100 \mu \mathrm{m}$, $\times 20$ field, and $50 \mu \mathrm{M}, \times 40$ field). Panel (B) shows the effects of treatment in CAF stained for $\alpha$ SMA (red) and vimentin (green) immunofluorescence for quantification of activated and total fibroblasts in untreated sample (left), sample treated with chemoradiation (middle) and sample treated with nab-paclitaxel and gemcitabine (right; $\times 40$; scale bar $=50 \mu \mathrm{m}$ ). Panel $(\mathbf{C})$ shows a quantification of total and activated CAFs in untreated samples, primary PDA tumours treated with chemoradiation, primary tumour PDA tumours treated with nab-paclitaxel and gemcitabine in two (20 steps) $\times 40 \mathrm{HCX}$ PL APO CS (1.25 NA) oil immersion objectives high-power fields. Samples treated with nab-paclitaxel and gemcitabine show a statistically significant lower activated CAF (Mann-Whitney tests were used for statistical analysis: ${ }^{\star} P=0.037$ ). Panel (D) represents the average CAF density index determined by dividing the number of CAF by proportion of stromal content in two $\times 40$ HCX PL APO CS (1.25 NA) oil immersion objectives high-power fields. As shown, nab-paclitaxel and gemcitabine-treated groups have a statistically significant lower activated CAF density (Mann-Whitney tests were used for statistical analysis: $\left.{ }^{\star} P=0.037 ;{ }^{\star \star} P=0.015\right)$.

GEMM of the disease. The study shows that nab-paclitaxel and gemcitabine was very effective inducing marked tumour responses by FDG-PET and CA19.9 levels. Seven of 10 patients with PDA had major pathological responses. Analysis of residual tissues indicated an important disruption of collagen architecture and a decrease in CAF, albeit the number of activated CAF remained unchanged. These effects translated into diminished tumour stiffness as measure by EUS elastography. In the co-clinical mouse study, we observed that nab-paclitaxel-treated animals have also a distorted collagen with low cellular content while gemcitabinetreated mice showed a marked increase in CAF.

Although the main objective of the study was not clinical outcome, and thus the sample size is limited to make any strong conclusion in that sense, the antitumour effects as shown by FDGPET, CA19.9 response, rate of R0 resections, and pathological responses, are notable and certainly support additional definitive studies in this setting (Chun et al, 2011; Andriulli et al, 2012). Of interest, and in agreement with the tissue-based analysis discussed below, we did not observe objective responses by RECIST. However, the majority of patients showed improvement in SUV uptake by FDG-PET and elastography ratio. This discrepancy between morphological and functional imaging is consistent with the tissue results and suggest that as a tumour respond to treatment there is a replacement of actively growing glucose consuming cells to an oligocellular, quiescent stroma. The lack of concordance between RECIST, metabolic, and pathological responses points to the need to incorporate other measurements of efficacy in preoperative studies in PDA. Three patients, however, develop rapid disease progression indicating that there is a group of patients who progress rapidly to nab-paclitaxel and gemcitabine. Biomarkers to identify these patients are of mayor interest.

One of the goals of this work was to incorporate new methods to evaluate stromal changes. To this end, we focused on EUS-based elastography. This imaging modality is currently used to determine the degree of liver fibrosis in patients with end-stage liver disease. In PDA, its main application has been the differential diagnosis of benign and malignant pancreas masses (Janssen et al, 2007; Iglesias-Garcia et al, 2010; Dawwas et al, 2012). In this study, we show that tumour stiffness decreased during treatment and that this effect correlated with improvement in CA19.9 and FDG uptake likely reflecting modification in the cancer stroma as shown in the tissue analysis. Elastography should be further studied in preoperative trials in PDA and in particular in those with agents targeting the stroma. 


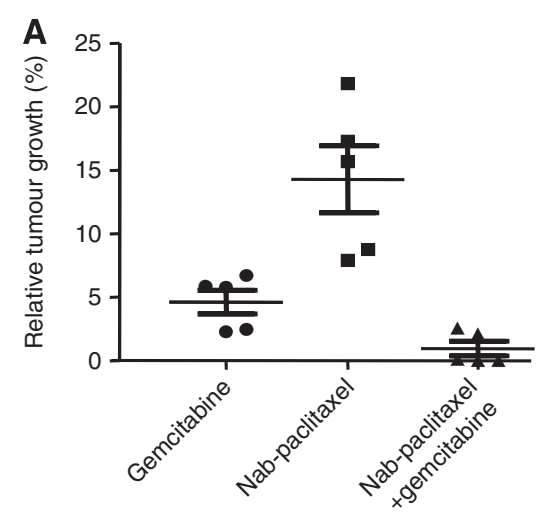

\section{B}
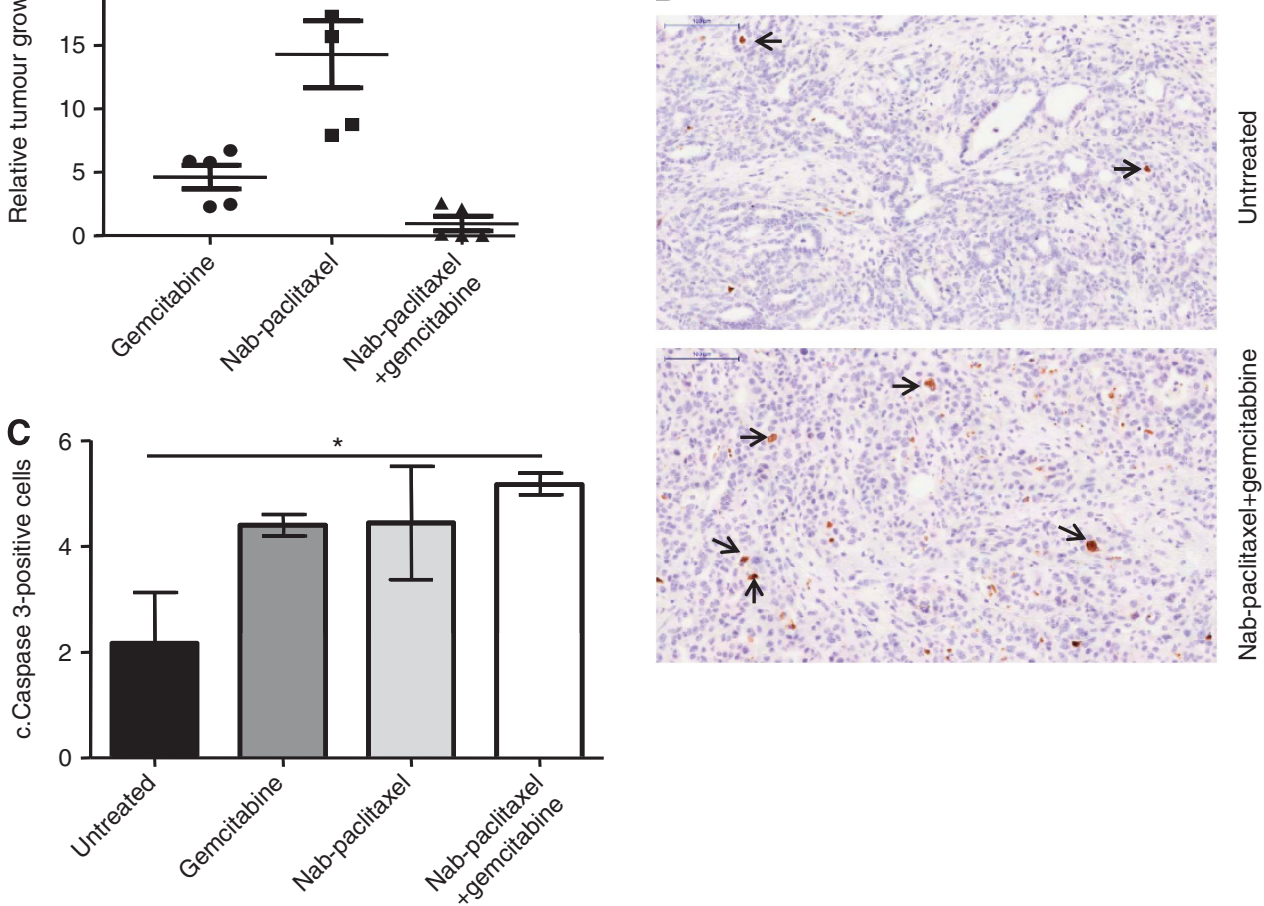

Figure 3. Antitumour effects of nab-paclitaxel and gemcitabine in GEMM of PDA. Panel (A) shows tumour growth inhibition of individual mouse treated with gemcitabine, nab-paclitaxel, or the combination. As depicted, the combined treatment was more effective than treatment with either gemcitabine alone $(P=0.01)$ or nab-paclitaxel $(P=0.001)$. Panel $(B)$ shows representative microphotograph of caspase 3 expression showing increased level of apoptosis in the treated tumours. Panel (C) shows the quantification of caspase 3-positive cells in the different treatment groups showing a statistically significant increased in the combination treated group compared with control ( $P=0.047$, assessed by Student's $t$-test related to the untreated group).

The effects of nab-paclitaxel in PDA stroma are not fully understood and to some extent controversial. In our prior studies in patient-derived xenograft mouse models treated for short period of time, we observed an almost complete elimination of cancer stroma (Von Hoff et al, 2011). These results, however, were not reproduced in studies using GEMM of PDA (Frese et al, 2012). Here we show, in patient samples, that the effect is predominantly stromal remodelling with marked alteration of collagen architecture and elimination of CAF. This is best reflected by changes in CAF density and appears to be different from patients treated with conventional chemoradiation. Similar observations were made in GEMM of PDA driven by distinct engineered genetic changes as the models tested before: the most striking observation was the increase collagen and CAF in the gemcitabine only treated group. The reasons for these differences across models and patients are not known. Duration of treatment can be a factor as mice studies are conducted with short, 5-21 days, exposure while in human studies the treatment duration is 8 weeks allowing time for some of the tissue remodelling effects to occur. Another factor is differences between models. Human-derived xenografts models form less stroma of murine origin and have abnormal tumour vascularisation, particularly in subcutaneous implanted tumours. In contrast, the KPC GEMM model used in prior studies forms abundant and exuberant stroma (Olive et al, 2009). The GEMM model used in these studies, carrying different engineered genetic changes, has an intermediate stromal content not as manifested as the KPC model.

There are, however, limitations to this work that need to be taken into account when considering the implications of these data.
The sample size is small and includes patients with different stages, even within a group of patients with localises PDA, making any conclusion hypothesis generating. Indeed, the content of stroma and CAF may change with different cancer stages. Another limitation is that the study does not include a pre- and posttreatment tissue biopsy to determine tissue changes but rather an untreated control. Finally, there is no randomised control arm treated with gemcitabine alone that would make the comparison more conclusive.

In summary, this clinical study shows that nab-paclitaxel plus gemcitabine exerts significant antitumour activity in primary PDA as seen by the high rate of responses by different imaging methods and tumour marker studies that led to marginfree resection in $92 \%$ of patients and pathological responses in $70 \%$ of cases. Although the number of patients is small, the results are striking enough to warrant additional studies in this setting. At the tissue level, the most interesting observation of the study is that, in marked contrast with tumour exposed preoperatively to conventional chemoradiation, tumours treated with nab-paclitaxel contained less abundant CAFs, a clearly altered collagen, and had became softer as determined by elastography. Parallel mouse studies indicate that the main effects are due to nab-paclitaxel and not gemcitabine. Based on these data, this approach should be tested in a larger randomise trial to determine the clinical impact of tumour down staging before surgery and the long-term impact of pathological tumour regression. In addition, EUS elastography appears an attractive method to monitor stromal changes with treatment. 

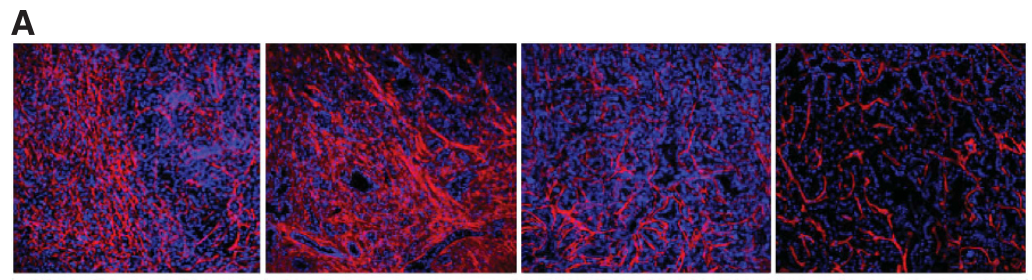

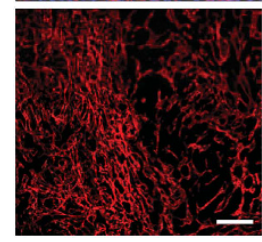

Control

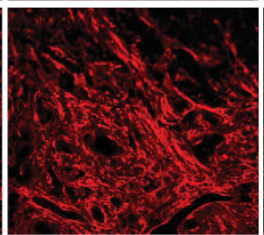

Gemcitabine

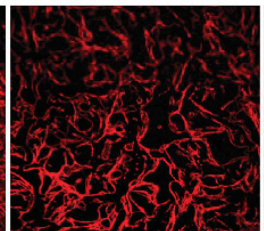

Nab-paclitaxel

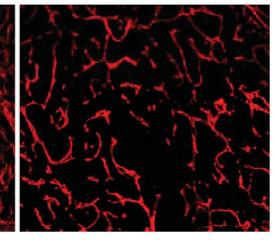

Nab-paclitaxel + gemcitabine

B

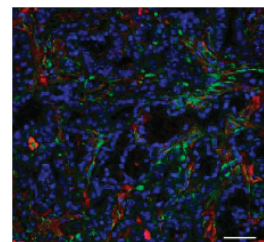

Control

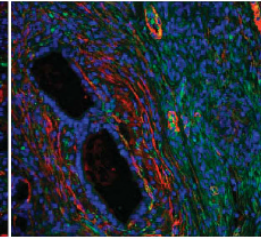

Gemcitabine

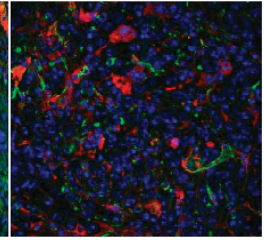

Nab-paclitaxel

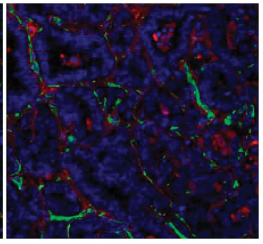

Nab-paclitaxel + gemcitabine

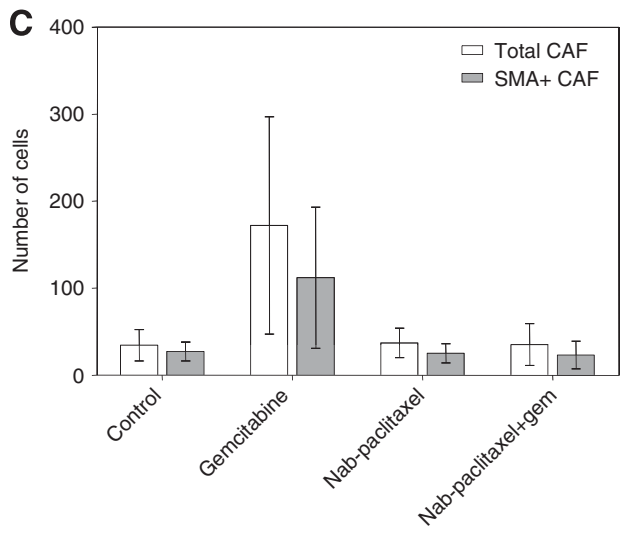

Figure 4. Tissue effects of treatment regimens in GEMM of PDA. Panel (A) shows confocal microscopy analysis of type I collagen immunofluorescence as main component of extracellular matrix in untreated tumours and tumours treated with gemcitabine, nab-paclitaxel, and the combination ( $\times 20$, top, and detail of collagen network at $\times 20$, bottom; scale bar $=75 \mu \mathrm{m}, \times 20$ field). Panel (B) shows the effects of treatment in CAF stained for $\alpha$ SMA (red) and vimentin (green) immunofluorescence for quantification of activated and total fibroblast in samples of mice treated with the indicated agents ( $\times 40$, scale bar $=50 \mu \mathrm{M}$ ). Panel $(\mathbf{C})$ shows a quantification of total and activated CAFs in untreated tumours and tumours treated with gemcitabine, nab-paclitaxel and the combination in two (20 steps) $\times 40 \mathrm{HCX}$ PL APO CS (1.25 NA) oil immersion objectives high-power fields. Statistical analysis was not significant because of heterogeneity of samples.

\section{ACKNOWLEDGEMENTS}

We thank JI Gordon (Washington University School of Medicine, St Louis, MO, USA) for providing the tetO-PhCMV-Cre mice and Eric Sandgren (University of Wisconsin, Madison, WI, USA) for providing the Elastase-tTA strain. This study was supported by the Fondo de Investigaciones Sanitarias (FIS) PI10-01996 and Celgene Inc. to MH; the European Research Council (ERC-AG/250297RAS AHEAD) and Spanish Ministry of Economy and Competitiveness (SAF2011-30173) to MB.

\section{CONFLICT OF INTEREST}

The authors declare no conflict of interest.

\section{REFERENCES}

Andriulli A, Festa V, Botteri E, Valvano MR, Koch M, Bassi C, Maisonneuve P, Sebastiano PD (2012) Neoadjuvant/preoperative gemcitabine for patients with localized pancreatic cancer: a meta-analysis of prospective studies. Ann Surg Oncol 19: 1644-1662.

Arnold SA, Rivera LB, Miller AF, Carbon JG, Dineen SP, Xie Y, Castrillon DH, Sage EH, Puolakkainen P, Bradshaw AD, Brekken RA (2010) Lack of host SPARC enhances vascular function and tumor spread in an orthotopic murine model of pancreatic carcinoma. Dis Model Mech 3: $57-72$.

Chun YS, Cooper HS, Cohen SJ, Konski A, Burtness B, Denlinger CS, Astsaturov I, Hall MJ, Hoffman JP (2011) Significance of pathologic response to preoperative therapy in pancreatic cancer. Ann Surg Oncol 18: 3601-3607.
Dawwas MF, Taha H, Leeds JS, Nayar MK, Oppong KW (2012) Diagnostic accuracy of quantitative EUS elastography for discriminating malignant from benign solid pancreatic masses: a prospective, single-center study. Gastrointest Endosc 76(5): 953-961.

Eisenhauer EA, Therasse P, Bogaerts J, Schwartz LH, Sargent D, Ford R, Dancey J, Arbuck S, Gwyther S, Mooney M, Rubinstein L, Shankar L, Dodd L, Kaplan R, Lacombe D, Verweij J (2009) New response evaluation criteria in solid tumours: revised RECIST guideline (version 1.1). Eur J Cancer 45: 228-247.

Erkan M, Michalski CW, Rieder S, Reiser-Erkan C, Abiatari I, Kolb A, Giese NA, Esposito I, Friess H, Kleeff J (2008) The activated stroma index is a novel and independent prognostic marker in pancreatic ductal adenocarcinoma. Clin Gastroenterol Hepatol 6: 1155-1161.

Frese KK, Neesse A, Cook N, Bapiro TE, Lolkema MP, Jodrell DI, Tuveson DA (2012) nab-Paclitaxel potentiates gemcitabine activity by reducing cytidine deaminase levels in a mouse model of pancreatic cancer. Cancer Discov 2: 260-269.

Guerra C, Collado M, Navas C, Schuhmacher AJ, Hernandez-Porras I, Canamero M, Rodriguez-Justo M, Serrano M, Barbacid M (2011) Pancreatitis-induced inflammation contributes to pancreatic cancer by inhibiting oncogene-induced senescence. Cancer Cell 19: 728-739.

Hidalgo M (2010) Pancreatic cancer. N Engl J Med 362: 1605-1617.

Iglesias-Garcia J, Larino-Noia J, Abdulkader I, Forteza J, Dominguez-Munoz JE (2010) Quantitative endoscopic ultrasound elastography: an accurate method for the differentiation of solid pancreatic masses. Gastroenterology 139: $1172-1180$

Infante JR, Matsubayashi H, Sato N, Tonascia J, Klein AP, Riall TA, Yeo C, Iacobuzio-Donahue C, Goggins M (2007) Peritumoral fibroblast SPARC expression and patient outcome with resectable pancreatic adenocarcinoma. J Clin Oncol 25: 319-325.

Jacobetz MA, Chan DS, Neesse A, Bapiro TE, Cook N, Frese KK, Feig C, Nakagawa T, Caldwell ME, Zecchini HI, Lolkema MP, Jiang P, Kultti A, Thompson CB, Maneval DC, Jodrell DI, Frost GI, Shepard HM, Skepper JN, Tuveson DA (2013) Hyaluronan impairs vascular function and drug delivery in a mouse model of pancreatic cancer. Gut 62(1): 112-120. 
Janssen J, Schlorer E, Greiner L (2007) EUS elastography of the pancreas: feasibility and pattern description of the normal pancreas, chronic pancreatitis, and focal pancreatic lesions. Gastrointest Endosc 65: 971-978.

Mahadevan D, Von Hoff DD (2007) Tumor-stroma interactions in pancreatic ductal adenocarcinoma. Mol Cancer Ther 6: 1186-1197.

Olive KP, Jacobetz MA, Davidson CJ, Gopinathan A, McIntyre D, Honess D, Madhu B, Goldgraben MA, Caldwell ME, Allard D, Frese KK, Denicola G, Feig C, Combs C, Winter SP, Ireland-Zecchini H, Reichelt S, Howat WJ, Chang A, Dhara M, Wang L, Ruckert F, Grutzmann R, Pilarsky C, Izeradjene K, Hingorani SR, Huang P, Davies SE, Plunkett W, Egorin M, Hruban RH, Whitebread N, McGovern K, Adams J, Iacobuzio-Donahue C, Griffiths J, Tuveson DA (2009) Inhibition of Hedgehog signaling enhances delivery of chemotherapy in a mouse model of pancreatic cancer. Science 324: 1457-1461.

Provenzano PP, Cuevas C, Chang AE, Goel VK, Von Hoff DD, Hingorani SR (2012) Enzymatic targeting of the stroma ablates physical barriers to treatment of pancreatic ductal adenocarcinoma. Cancer Cell 21: $418-429$.
Ryan R, Gibbons D, Hyland JM, Treanor D, White A, Mulcahy HE, O’Donoghue DP, Moriarty M, Fennelly D, Sheahan K (2005) Pathological response following long-course neoadjuvant chemoradiotherapy for locally advanced rectal cancer. Histopathology 47: 141-146.

Von Hoff DD, Korn R, Mousses S (2009) Pancreatic cancer-could it be that simple? A different context of vulnerability. Cancer Cell 16: 7-8.

Von Hoff DD, Ramanathan RK, Borad MJ, Laheru DA, Smith LS, Wood TE, Korn RL, Desai N, Trieu V, Iglesias JL, Zhang H, Soon-Shiong P, Shi T, Rajeshkumar NV, Maitra A, Hidalgo M (2011) Gemcitabine plus nabpaclitaxel is an active regimen in patients with advanced pancreatic cancer: a phase I/II trial. J Clin Oncol 29: 4548-4554.

This work is published under the standard license to publish agreement. After 12 months the work will become freely available and the license terms will switch to a Creative Commons AttributionNonCommercial-Share Alike 3.0 Unported License.

Supplementary Information accompanies this paper on British Journal of Cancer website (http://www.nature.com/bjc) 